\title{
MICROWAVE PROCESSING OF FOOD SAMPLES: INFLUENCE OF CAVITY DESIGN AND DIELECTRIC PROPERTIES
}

\author{
C. D. Albuquerque, S. Curet, L. Boillereaux \\ UMR GEPEA CNRS 6144 - ONIRIS, CS 82225, 44322 Nantes-France \\ sebastien.curet@oniris-nantes.fr
}

Keywords: microwave heating, modelling, impedance matching, temperature distribution.

\begin{abstract}
Microwave-material interactions and electromagnetic propagation phenomena are important to optimize the microwave heating process of food samples. In this study, a 3D numerical modelling of a TE10 rectangular waveguide including microwave antenna and impedance matching elements is proposed. The microwave applicator is aimed to process both solid and liquid food samples. The model illustrates the standing wave patterns and microwave absorbed power within the cavity by taking into account the influence of the screw tuner, quartz windows, shorting plunger, and additional dielectric support plates.

The results reveal the importance to consider the real cavity design and the precise dielectric characterization to predict accurate temperature profiles within the food product during the microwave heating. Such a model can be now be used to optimize the food sample geometry to achieve minimum reflected power and better heating uniformity.
\end{abstract}

\section{Introduction}

Microwave heating processes for food products are used industrially for various unit operations such as tempering, drying, pasteurization ${ }^{1}$. During a microwave heating process, the energy contained into an electromagnetic wave is converted into heat within dielectric materials. This selective volumetric heating mechanism allows a considerable reduction in processing time and increases the energy efficiency of the process ${ }^{2}$. However, one of the main challenges is the heterogeneous electric field distribution within the food matrix which could result in hot and cold spots ${ }^{3}$. Since electromagnetic field measurements within a microwave cavity are particularly difficult to perform, the numerical modeling and simulation appear as a promising tool to understand, predict and control the process, as well as, to optimize the design of equipment and packaging ${ }^{4}$. As many factors influence the degree of interaction between the microwaves and the material, the model needs to take into account both geometrical details of the microwave cavity and the physical properties of the materials under consideration. These include dielectric, thermal and physical properties of the product and the dielectric materials (volume, geometry and location) and the design of the microwave cavity ${ }^{5}$. The purpose of this study is to investigate the influence of the cavity design and dielectric properties during the microwave processing of solid and liquid food products.

\section{Design of the microwave cavity}

A solid-state microwave generator (SG 524, model AG340M3, Alter, Reggio Emilia, Italy) enables to supply a monochromatic wave transmitted along the $z$-direction of a TE10 rectangular waveguide (cross-section $86 \mathrm{~mm} \times 43 \mathrm{~mm}$ ) at a frequency of $2.45 \mathrm{GHz}$ with adjustable output power. The device includes an antenna placed at the waveguide transition, a 3-stub tuner with adjustable screws, two quartz windows to protect the upper and lower sides of the microwave applicator, a liquid food product flowing continuously within a 
quartz tube and a shorting plunger. Impedance matching is important to insure maximum transfer of microwave power from the microwave generator to the dielectric

loads. In this work, both the screw tuners and the waveguide shorting plunger are used to perform impedance matching between the waveguide and the dielectric samples by reducing the microwave reflected power. The experimental device is detailed in Fig. 1.
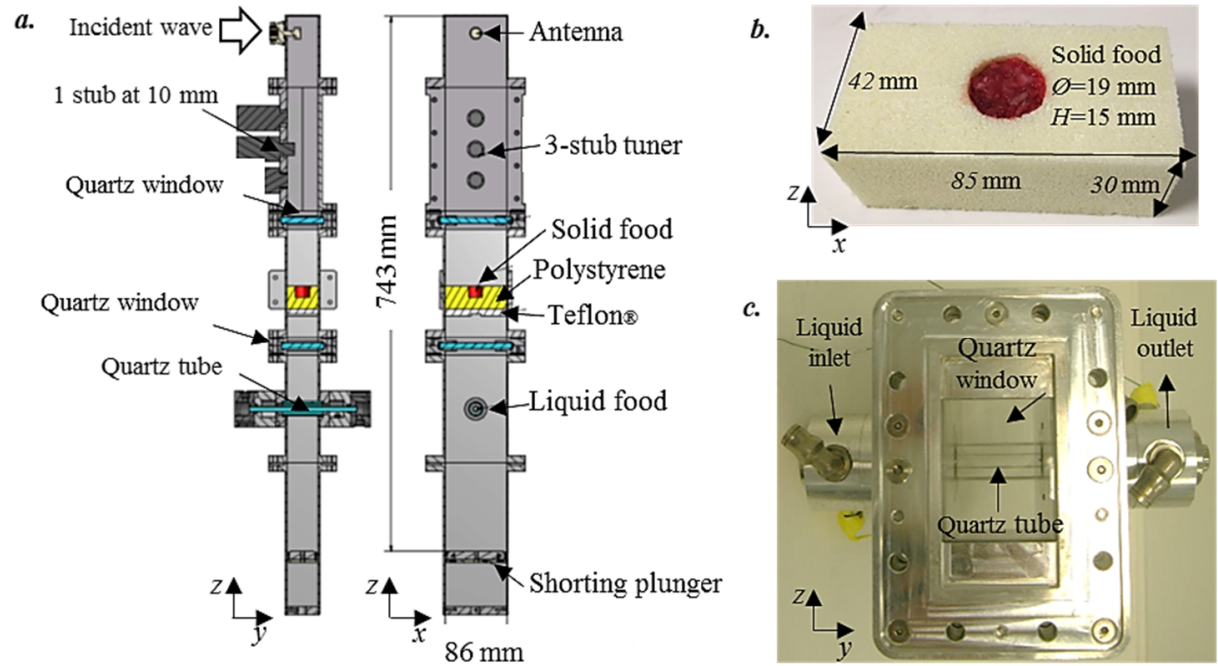

Fig. 1 (a) Schema of the microwave experimental apparatus (b) solid food product within a polystyrene block (c) quartz window and liquid food within a quartz tube.

The microwave applicator consists in $5 \mathrm{~g}$ of solid food sample and a continuous flow of liquid food within a quartz tube $(6 \mathrm{~mm}$ inlet diameter, $2 \mathrm{~mm}$ thick, liquid flowrate $300 \mathrm{~L} / \mathrm{h})$. The quartz tube crosses the small side of the rectangular waveguide (Fig 1c).

To improve thermal insulation of the solid food sample, the product was inserted on a cylindrical orifice (19 $\mathrm{mm}$ diameter, $15 \mathrm{~mm}$ length) in the centre of the polystyrene block (85 mm x $42 \mathrm{~mm} \times 30 \mathrm{~mm}$ ) filling the cross-section of the waveguide (Fig 1b). In addition, a Teflon ${ }^{\circledR}$ support plate is inserted at the bottom of the polystyrene block. The temperature is measured at two locations within the sample with sheathed type-K thermocouples. Temperatures are recorded by a data logger (OM-CP QuadTemp 2000, OMEGA, France). Impedance matching conditions are reached by setting one screw displacement at $10 \mathrm{~mm}$ for a full cavity length of $743 \mathrm{~mm}$ (Fig 1a).

\section{Model design}

The real geometry of the experimental device is considered for the $3 \mathrm{D}$ numerical modelling. The model is solved using the $\mathrm{COMSOL}^{\circledR}$ Multiphysics 5.3a software which uses finite element method for discretization of equations. Mesh refinements is performed at narrow regions of the geometry to insure a sufficient numerical precision. The selected mesh size consisted in a total of 1.000 .760 tetrahedral elements to obtain the mesh independence of numerical results (Fig. 2). 


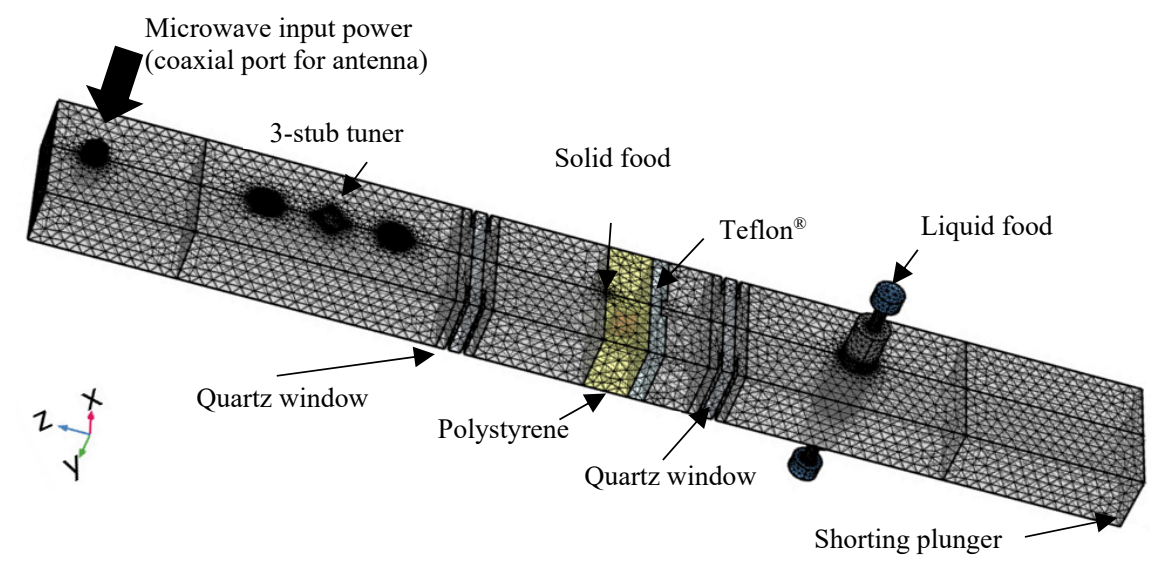

Fig. 2 3D numerical modelling of the full microwave rectangular waveguide

The model is aimed at predicting both electric field distributions in the TE10 mode rectangular waveguide and temperature profiles in a solid food product. To achieve this objective, the following assumptions are introduced:

(i) The initial temperature of the food sample is uniform;

(ii) The processed solid food sample is considered as a homogeneous isotropic cylinder with constant thermophysical properties;

(iii) The dielectric properties of the materials are constant;

(iv) The shrinkage and mass transfer of the solid food sample are negligible;

(v) The upper surface of the solid sample is surrounded by air (zero dielectric losses).

The Maxwell's equations are solved numerically to predict the electromagnetic field distribution into the rectangular waveguide. Input microwave power is supplied at the antenna via a coaxial port $(20 \mathrm{~W})$. External waveguide walls are considered as perfect electric conductors to propagate a standing wave within the waveguide.

The general equation for electric field propagation within a material is defined as follows ${ }^{6}$ : $\nabla^{2} E+\omega^{2} \mu \varepsilon\left(1-j \frac{\sigma}{\omega \varepsilon}\right) E=0 \quad$ with $\varepsilon=\varepsilon_{0} \varepsilon_{r}^{\prime}-j \varepsilon_{0} \varepsilon^{\prime \prime}{ }_{r}$ and $\mu=\mu_{0} \mu_{r}$

Where $\square{ }^{\prime} r$ is the relative dielectric constant of the material, $\square{ }^{\prime}{ }_{r}$ the loss factor, $\square 0$ the permittivity of a vacuum, $\mu_{r}$ the relative permeability and $\mu_{0}$ the permeability of a vacuum. The dielectric properties of the various materials are depicted in Table 1.

The internal heat generation term $\left(Q_{a b s}\right)$ quantifies the amount of power dissipated into the material by dielectric losses. The heat generation term is computed from the local electric field strength at any location of the sample domain from the resolution of the Maxwell's equations.

$$
Q_{a b s}=\frac{1}{2} \omega \varepsilon_{0} \varepsilon_{r}^{\prime \prime}\left|E_{\text {local }}\right|^{2}
$$

For a solid food product and a heat generation due to microwaves, the classical heat transfer equation without any convection term is reduced to:

$$
\rho C_{p} \frac{\partial T}{\partial t}=\operatorname{div}(k \nabla T)+Q_{a b s}
$$


This equation depends on the thermophysical properties of the food sample. The specific heat $\left(3.69 \mathrm{~kJ} \mathrm{~kg}^{-1} \mathrm{~K}^{-1}\right)$ and density $\left(1130 \mathrm{~kg} / \mathrm{m}^{3}\right)$ were determined experimentally and thermal conductivity $\left(0.41 \mathrm{~W} \mathrm{~m}^{-10} \mathrm{C}^{-1}\right)$ was taken from literature for meat sample ${ }^{7}$. The upper surface of the food product is submitted to an external convective heat flux expressed as a boundary condition:

$$
k . \nabla T=h_{\text {air }} \cdot\left(T-T_{\infty}\right), \quad \forall(x, y, z), \forall t>0, \quad T_{\infty}=20^{\circ} \mathrm{C}
$$

The convective heat transfer coefficient was estimated experimentally $\left(6.5 \mathrm{~W} / \mathrm{m}^{2} / \mathrm{K}\right)$ by the lumped capacitance method.

Table 1. Relative dielectric constant $\left(\square r^{\prime}\right)$ and dielectric loss factor ( $\square r$ '”) obtained experimentally by resonant cavity technique (polystyrene, Teflon ${ }^{\circledR}$ ) and open-ended coaxial probe for solid and liquid food samples), for each material at $20^{\circ} \mathrm{C}$. Dielectric properties for quartz were taken from literature ${ }^{8}$.

\begin{tabular}{lcccccc}
\hline $\begin{array}{l}\text { Dielectric } \\
\text { properties }\end{array}$ & Air & Solid food & Polystyrene & Quartz & Teflon ${ }^{\circledR}$ & Liquid food \\
\hline$\square{ }^{\prime}{ }_{r}$ & 1 & 40.3 & 1 & 4.2 & 1.7 & 77.6 \\
$\square{ }^{r}$ & 0 & 15.1 & $2.510^{-5}$ & 0 & $3.410^{-4}$ & 12.3 \\
\hline
\end{tabular}

The model is solved numerically as a two-way coupling: the electromagnetic field distribution is firstly computed within the whole geometry following a harmonic propagation at $2.45 \mathrm{GHz}$. Then, the transient heat equation is considered to compute the temperature distribution within the solid food sample by considering the microwave absorbed power.

The model is solved with a Dell ${ }^{\circledR}$ Precision ${ }^{\mathrm{TM}}$ Workstation computer, equipped with $2 \times$ Intel $^{\circledR}$ Xeon processors ( 8 cores), at $2.5 \mathrm{GHz}$, with $256 \mathrm{~GB}$ of RAM, running on Windows ${ }^{\circledR} 8$ Professional, 64 bits.

Four scenarios are considered to investigate both the dielectric properties influence of materials and the cavity design on the electric field distribution.

Scenario 1: Waveguide transition with an antenna (coaxial port), solid and liquid food, and shorting plunger. Dielectric properties of other materials are assumed to be close to air; Scenario 2: Scenario 1 with the addition of one screw tuner with $10 \mathrm{~mm}$ displacement; Scenario 3: Scenario 2 by considering the dielectric properties of the quartz material; Scenario 4: Scenario 3 by considering dielectric properties of polystyrene and Teflon ${ }^{\circledR}$.

Following the four previous scenarios, both electric field distribution and temperature map within the solid food sample are investigated. To take into account the uncertainty of the temperature probe locations in the $(\mathrm{x}, \mathrm{z})$ plane, an experimental position error of $\pm 1 \mathrm{~mm}$ is considered at the two probe locations.

\section{Results and discussion}

For each considered scenario, a power balance is performed to compute the microwave forward, reflected and absorbed power within the dielectric samples.

For the first scenario without the screw tuner modelling, the cavity is not adapted $(64 \%$ of the forward power is reflected). By taking into account the real design of the cavity 
(scenario 4), the reflection coefficient is reduced to a minimum value of $9 \%$ (ratio of reflected power versus microwave input power). This value is found in agreement with experimental data.

For each scenario, a power balance is realized and the values are presented in Table 2.

Table 2. Power balance within the system for a microwave input power of $20 \mathrm{~W}$.

\begin{tabular}{cccc}
\hline Scenario & $\begin{array}{c}\text { Reflected } \\
\text { power }\end{array}$ & $\begin{array}{c}\text { Absorbed power within } \\
\text { the solid food sample }\end{array}$ & $\begin{array}{c}\text { Absorbed power within } \\
\text { the liquid food sample }\end{array}$ \\
\hline 1 & 13 & 3.6 & 3.4 \\
2 & 10 & 5 & 5 \\
3 & 6 & 2.4 & 11.5 \\
4 & 1.8 & 4 & 14.1 \\
\hline
\end{tabular}

Results indicates that impedance matching increases the energy efficiency of the system by reducing the reflected power $(13 \mathrm{~W}$ vs. $1.8 \mathrm{~W})$ and by increasing the absorbed power within the liquid sample $(3.4 \mathrm{~W}$ vs. $14.1 \mathrm{~W})$. However, the solid food sample absorbs different microwave power depending on the design of the equipment. The modelling of the screw tuner clearly influences the predicted electric field distribution. The quartz, polystyrene and Teflon $^{\circledR}$ materials are known as microwave-transparent materials due to their very low dielectric loss factor. Nevertheless, these materials have a non-negligible dielectric constant value (Table 2). Thus, they are able to polarize the electric field resulting in modification of the electric field distribution within the waveguide, as illustrated in Fig. 3.

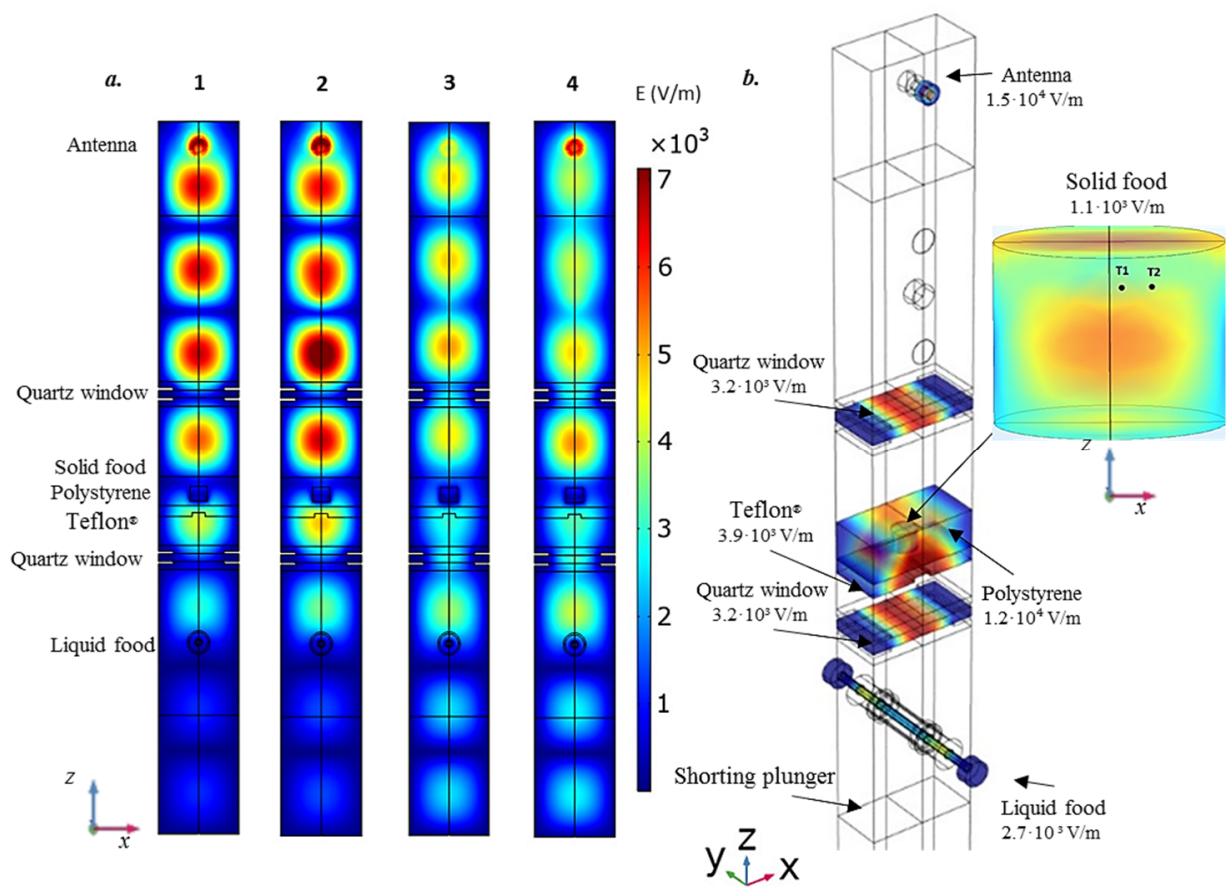

Fig. 3 (a) Electric field distribution within the rectangular waveguide (b) Electric field patterns within dielectric materials with maximum electric field strength values. 
Under our operating conditions, the incident wave is superimposed with the reflected wave which leads to a standing wave pattern. For each scenario, the electric field distribution is displayed on a line located at the center of the rectangular waveguide and along the microwave propagation direction (from the antenna to the short plunger location).
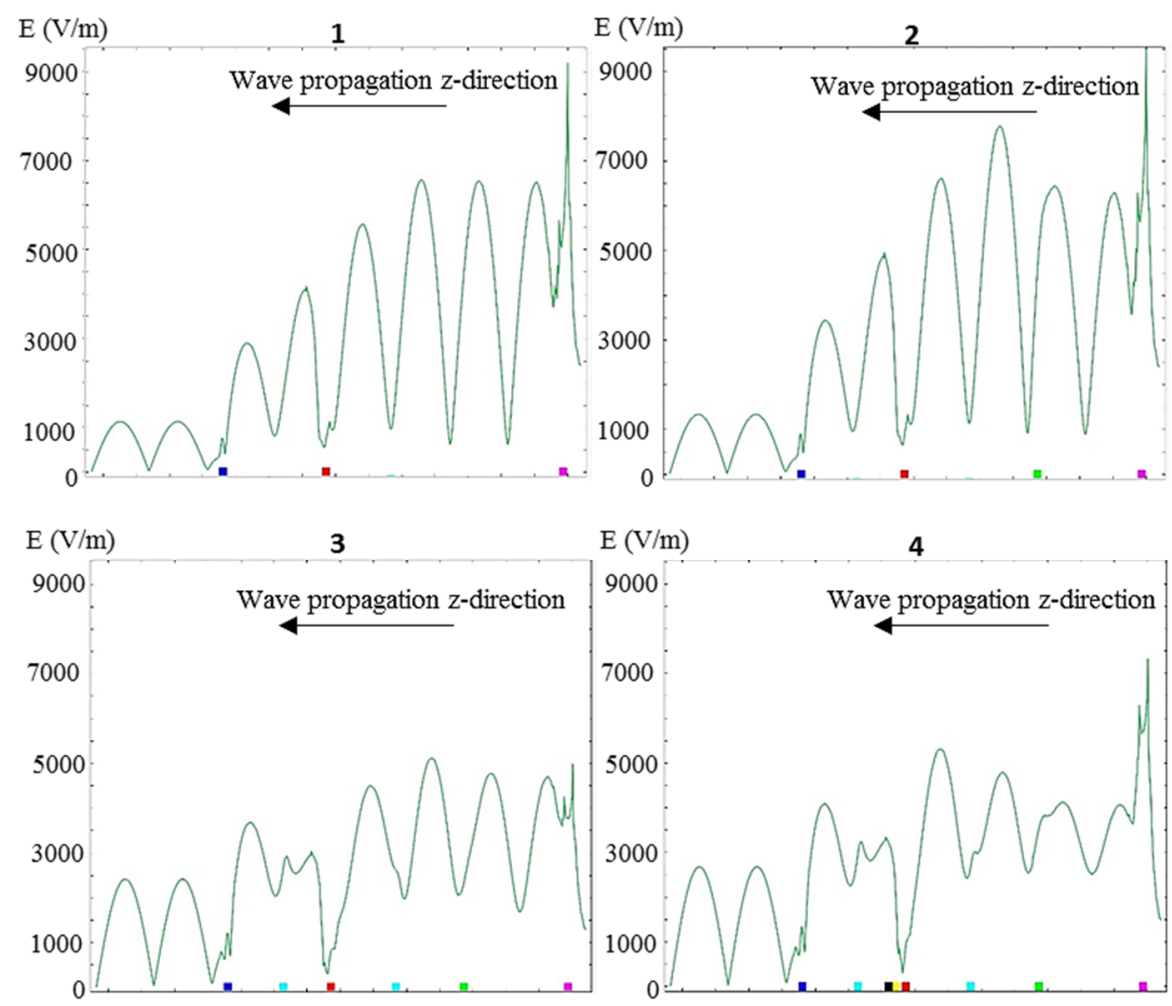

Subtitle

$\square$ Liquid food $\square$ Teflon॰ Polystyrene $\square$ Solid food $\square$ Quartz window $\square$ Stub $\square$ Antenna

Fig. 4 Standing wave patterns for each scenario

Fig. 4 clearly illustrates the influence of the antenna, screw tuner, quartz, polystyrene and Teflon ${ }^{\circledR}$ materials on the electric field distribution. Due to its small size, the solid food sample is always located at a region where the electric field strength is minimal.

Temperature distribution with the solid food matrix is then evaluated by considering a microwave heating process at $20 \mathrm{~W}$ during 131 seconds. Experimental temperature measurements at two locations are compared with simulated temperature according to the four previously detailed scenarios (Fig. 5). The numerical simulation clearly indicates a heterogeneous temperature distribution within the solid food sample, with the presence of hot and cold spots for all four scenarios. For each case, a hot spot is found in the upper center and a cold spot in the lower side of the sample, as shown in the Fig. 5. In the second scenario (Fig. 5-2), the solid food sample absorbs more microwave power (5W, Table 2), resulting in a large temperature gradient within the sample volume. The temperature in the hot spot reaches $82^{\circ} \mathrm{C}$ and in the cold spot only $29^{\circ} \mathrm{C}$. 

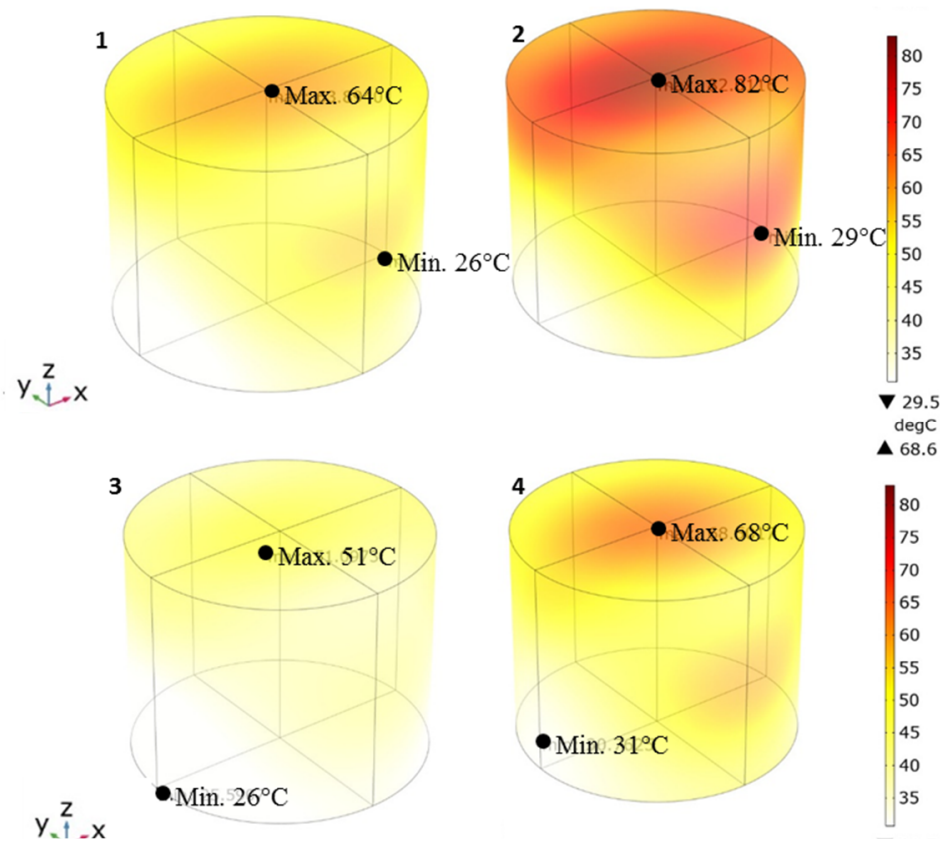

Fig. 5 3D temperature distribution in the solid food sample for each scenario.
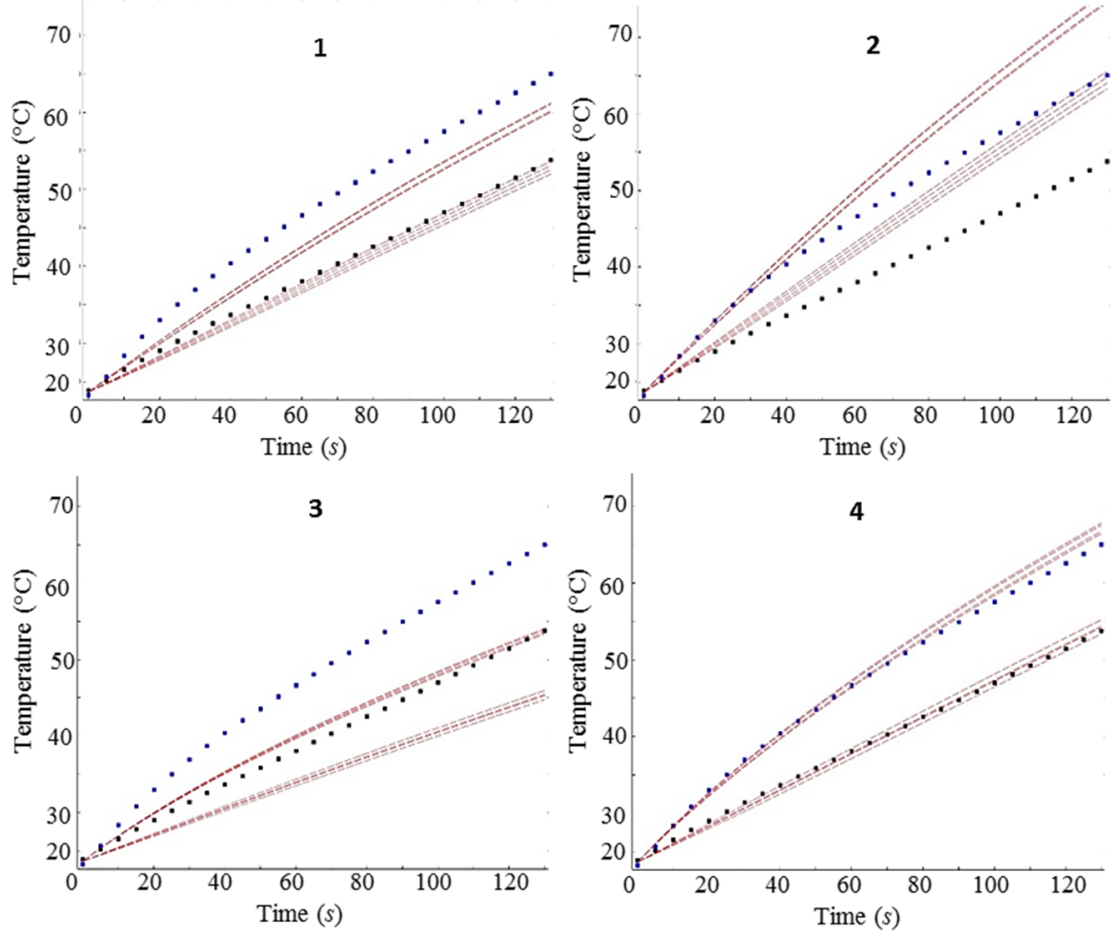

Fig. 6 Experimental vs. predicted temperatures. Red broken lines represent the simulated temperatures at two locations with probe position uncertainties and blue (T1) and black (T2) diamonds represent the experimental data. 
For scenario 1 and 3, the temperature measured experimentally is always higher than the simulated ones. For scenario 4, the simulation results show a good correlation with the experimental data (Fig. 6-4) when all the geometrical details of the rectangular waveguide are taken into account for the 3D numerical modelling.

\section{Conclusion}

In this study, a 3D numerical modelling of a microwave heating process in a single-mode rectangular waveguide is performed. The results are analyzed following different scenarios to illustrate the influence of the cavity design and dielectric properties of tested materials on both the absorbed power and temperature distribution within food samples. In the case of a standing wave pattern inside a microwave cavity, this study demonstrates the importance to consider all the geometrical details of the cavity and the accurate dielectric properties characterization of the materials to predict the electric field distribution. The proposed methodology could now be extended to design a single mode microwave applicator with optimized geometry to reduce temperature gradients inside the food matrix using external heat sources, such as infrared or hot air.

\section{Acknowledgements}

The authors are grateful to the National Council for Scientific and Technological Development (CNPq, Brazil) for Clarissa Detomi Albuquerque's PhD scholarship and financial support (process number 232767/2014-9). The authors would also like to thank O. Tantot (XLIM, UMR CNRS 7252) for the dielectric characterization of materials.

\section{References}

[1] Ahmed, J. \& Ramaswamy, H. Microwave Pasteurization and Sterilization of Foods. in Handbook of Food Preservation (ed. Taylor \& Francis Group, L.) 691711 (2007). doi:10.1201/9781420017373.ch28

[2] Kostas, E. T., Beneroso, D. \& Robinson, J. P. The application of microwave heating in bioenergy: A review on the microwave pre-treatment and upgrading technologies for biomass. Renew. Sustain. Energy Rev. 77, 12-27 (2017).

[3] Motasemi, F. \& Afzal, M. T. A review on the microwave-assisted pyrolysis technique. Renew. Sustain. Energy Rev. 28, 317-330 (2013).

[4] Pitchai, K., Birla, S. L., Subbiah, J., Jones, D. \& Thippareddi, H. Coupled electromagnetic and heat transfer model for microwave heating in domestic ovens. J. Food Eng. 112, 100-111 (2012).

[5] Zhang, H. \& Datta, A. K. Coupled electromagnetic and thermal modeling of microwave oven heating of foods. J. Microw. Power Electromagn. Energy 35., $71-85$ (2000).

[6] Curet, S., Rouaud, O. \& Boillereaux, L. Effect of Sample Size on Microwave Power Absorption Within Dielectric Materials: 2D Numerical Results vs. ClosedForm Expressions. AIChE J. 55, 1569-1583 (2009).

[7] Pan, Z. \& Paul Singh, R. Physical and thermal properties of ground beef during cooking. LWT - Food Sci. Technol. 34, 437-444 (2001).

[8] Salema, A. A. \& Afzal, M. T. Numerical simulation of heating behaviour in biomass bed and pellets under multimode microwave system. Int. J. Therm. Sci. 91, 12-24 (2015). 